\title{
BMJ Open Patients' experiences of a multidisciplinary team-led community case management programme: a qualitative study
}

\author{
Alice Gowing, Claire Dickinson, Tom Gorman, Louise Robinson, Rachel Duncan
}

To cite: Gowing A, Dickinson C, Gorman T, et al. Patients' experiences of a multidisciplinary team-led community case management programme: a qualitative study. BMJ Open 2016;6:e012019. doi:10.1136/bmjopen-2016012019

- Prepublication history and additional material is available. To view please visit the journal (http://dx.doi.org/ 10.1136/bmjopen-2016012019).

Received 29 March 2016 Revised 24 May 2016 Accepted 17 August 2016

CrossMark

Institute of Health and Society, Newcastle University, Newcastle upon Tyne, UK

Correspondence to Dr Rachel Duncan; r.duncan@ncl.ac.uk

\section{ABSTRACT}

Objectives: To explore the views and experiences of patients on the care they have received while enrolled on the Northumberland High Risk Patient Programme (NHRPP). This programme involved case finding of frail patients using a multidisciplinary team (MDT)-led community case management programme, and support of patients through care planning and regular reviews using primary, community, secondary and social care professionals.

Design: A qualitative study using semistructured interviews, which were digitally recorded, transcribed and subject to thematic analysis.

Setting: Community patients receiving primary care in the county of Northumberland, England.

Participants: 23 participants took part, of which 16 were patients enrolled on the NHRPP, and 7 carers. GP practices were selected purposively by size, deprivation and location, and patients identified and invited by General Practitioners to participate.

Results: 4 main themes emerged from the data: awareness and understanding of the NHRPP, confidence in the primary healthcare team, limitations of home care and the active role of being a patient. Despite having a low level of awareness of the details of the NHRPP, participants did think that its broad aim made sense. Participants discussed their high level of satisfaction with their care and access to team members. However, some limitations of alternatives to hospital care were identified, including the need to consider psychological as well as medical needs, the importance of overnight care and the needs of those without informal carers. Finally, participants discussed the active nature of being a patient under the NHRPP if they were to contribute fully to planning and managing their own care.

Conclusions: This study has identified that a programme of MDT-led case management was generally very well received by patients and their families. However, a number of factors were identified that could improve the implementation of the programme and further research needs to be undertaken to address these.
Strengths and limitations of this study

- This study fulfils calls for development of research around multidisciplinary team (MDT)-led community case management.

- While many similar studies to date have focused on care provided by an individual case manager, this study explored views on a programme facilitated by an MDT.

- This qualitative study allowed the indepth exploration of the experiences of patients enrolled on a local MDT-led case management programme.

- Although efforts were made to achieve a varied sample in terms of practice characteristics and participant demographics, the sample size was small and limited to one county of England.

\section{INTRODUCTION}

Populations are ageing globally, ${ }^{1}$ and the UK is no exception, ${ }^{2}$ with $80 \%$ of those over 80 years thought to have two or more longterm conditions ${ }^{3}$ with an estimated $25-50 \%$ of people over 85 years classed as frail. ${ }^{4}$ The increasing burden that multimorbidity and frailty places on health systems ${ }^{56}$ has led policymakers and academics internationally to seek to address this. ${ }^{67}$

Frail, multimorbid elderly people are best served by a system which integrates health and social care with emphasis on optimising chronic disease management and coordinating the complex services surrounding them. ${ }^{89}$ Case management is one model for providing this coordinated care and is defined as a targeted, community-based and proactive approach to care. $^{10}$

Significant research has focused on individual case manager-led programmes. ${ }^{11}{ }^{12}$ Some success has been shown in terms of cognitive function, physical health, psychological wellbeing, medication management, addressing 
unmet needs and hospital admission rates; however, these effects have not been found consistently and the evidence is often weak. ${ }^{12-15} \mathrm{~A}$ recent meta-analysis showed no significant impact on total cost of care or usage of secondary care, but did report a significant improvement in patient satisfaction. ${ }^{16}$

In the UK, recent efforts have been focused on case management led by multidisciplinary teams (MDTs). ${ }^{17} 18$ Evaluation of this approach has demonstrated little or no reduction in secondary care use; however, evidence of additional benefits for patients and professionals have begun to emerge, with calls for further investigation. ${ }^{19}$

The need for evaluation of case management programmes at a local level to understand barriers and facilitators to its effectiveness to inform new initiatives is well recognised and called for by the authors of previous studies. ${ }^{16}$ The Medical Research Council recommend qualitative methods are needed to gain a comprehensive understanding of the mechanisms involved in complex interventions. $^{20}$

In England, case management has recently been incentivised with the introduction of a primary care Avoiding Unplanned Admissions Enhanced Service (AUAES) in 2014. ${ }^{21}$ The Northumberland High Risk Patient Programme (NHRPP) is an example of case management led by MDTs in primary care which was introduced across Northumberland in 2012, and was modified in 2014 with the introduction of the AUAES. ${ }^{21}$ The specifications of this local Northumberland scheme are summarised in box 1. In the case of the NHRPP, case management is provided by an MDT with a broad skill mix rather than a single individual. Patients enrolled on it are those felt to be at high risk of unplanned hospital admission by health and social care professionals, and as such is not disease-specific. Its aim is to provide proactive, holistic and coordinated care to improve the health and well-being of patients in addition to reducing the risk of avoidable emergency hospital admissions.

Box 1 Summary of the key components of the Northumberland High Risk Patient Programme (NHRPP)

Identification of a minimum of $2 \%$ of the practice patient population at high risk of unplanned hospital admission-kept as a high-risk register (introduced in 2014 by AUAES)

- Assignment of a named GP to each patient

- Nurse-led screening assessments (based on geriatric screening assessment) \pm GP-targeted medical assessments where appropriate

- Development of a personalised care plan with each patient, including advanced care plans

- Regular multidisciplinary team (including social care) review of patients on the NHRPP

- Prompt follow-up of patients following discharge from hospital (within 3 days)

- Support from secondary care health professionals, for example, geriatricians and pharmacist
The aim of this study was to explore, using qualitative methods, the experiences of patients and their carers of this arrangement of case management as delivered by the NHRPP.

\section{METHODS}

Design

A qualitative study using semistructured interviews was conducted in the county of Northumberland, England.

\section{Sampling and recruitment}

Recruitment was a two-stage process. Initially, as independent researchers, we purposively sampled ${ }^{22}$ the 45 GP practices across Northumberland participating in the NHRPP. To ensure a range of patients who experience different primary care settings, we took into account practice size, practice deprivation score and geographical location. In total, 11 practices were enrolled in the study. In the second stage, individual GPs used convenience sampling to select potential participants who fulfilled the following criteria: enrolled on NHRPP, living in their own home, mental capacity to consent to participate and not in receipt of palliative care. Recruitment continued until data saturation was reached.

Potential participants were initially approached by a GP known to them who sent an invitation letter and participant information leaflet, and followed this up with a telephone call if there was no response at 2 weeks. The details of those interested in participating were passed to the research team who then telephoned to arrange an interview at a convenient time and location. All patients were given the option of having a self-defined carer present for the interview. They were reassured that interviews were confidential and voluntary, and that data would be anonymised.

\section{Data collection}

All interviews were conducted by AG, an academic GP trainee, between February and August 2015. Written informed consent was obtained from participants (patients and carers) prior to interview. Semistructured interviews were conducted in participants' own homes. AG was aware of the influence that personal characteristics of the interviewer may have on the collection and interpretation of qualitative data. ${ }^{23}$ She was open with participants about her role as an academic GP trainee and emphasised her independence from the NHRPP.

A topic guide was developed from the literature and in discussion with the study team. Broad headings were used to allow flexibility to explore issues in depth with participants and for questions to evolve as new themes emerged (see online supplementary appendix 1). All interviews were audio-recorded and transcribed verbatim by an external transcription agency. All transcripts were checked by AG and anonymised prior to analysis, and stored securely at Newcastle University. 


\section{Data analysis}

Transcripts were analysed after each interview using thematic analysis. ${ }^{24}$ Thematic analysis was chosen because it offers flexibility, can summarise key features of a large data set while also offering 'thick description' and allow for social as well as psychological interpretation of the data. ${ }^{24}$ All transcripts were read by the research team to gain familiarity with the data and then a coding framework was developed and built on as the team worked through the data set. Themes and subthemes were generated led by AG and CD, and refined in team meetings, where deviant cases were also identified. NVivo (V.10) software was used for data management. In the Results section, PT signifies quotes from patients and $\mathrm{C}$ from carers, with patient and carer numbers corresponding.

Members of the research team had diverse backgrounds representing primary care, secondary care and social care. Two members were academic GP trainees (AG and TG), one was a GP (LR), one a secondary care rheumatologist (RD) and one a qualified social worker and chartered psychologist (CD). The team were conscious of their personal and professional biases and reflected throughout data collection and analysis to improve the credibility of the data.

\section{RESULTS}

\section{Participants}

A total of 23 participants took part in the study; 16 patients and 7 family members. Table 1 shows the characteristics of patients participating. The majority of participants were women; 11 patients and 6 family members. Patients were aged between 48 and 90 (median age 81.5 years). A total of 11 patient participants lived alone and 5 lived with a spouse. Four main themes emerged from the data: awareness and understanding of the NHRPP, confidence in the primary

\begin{tabular}{lllll}
\multicolumn{4}{l}{ Table 1} & \multicolumn{3}{l}{ Patient participant characteristics } \\
\hline PT & & & Living & Carer present at \\
ID & Gender & Age & situation & interview \\
\hline 01 & Male & 76 & Spouse & Yes (wife) \\
02 & Female & 80 & Alone & No \\
03 & Female & 85 & Spouse & Yes (husband) \\
04 & Male & 76 & Spouse & Yes (wife) \\
05 & Female & 85 & Alone & Yes (daughter) \\
06 & Male & 77 & Spouse & Yes (wife) \\
07 & Female & 83 & Alone & No \\
08 & Female & 81 & Alone & No \\
09 & Male & 82 & Alone & No \\
10 & Female & 90 & Alone & No \\
11 & Female & 63 & Alone & No \\
12 & Male & 75 & Spouse & Yes (wife) \\
13 & Female & 48 & Alone & No \\
14 & Female & 90 & Alone & No \\
15 & Female & 87 & Alone & No \\
16 & Female & 84 & Alone & Yes (daughter) \\
\hline
\end{tabular}

healthcare team, limitations of home care and the active role of being a patient.

\section{Awareness and understanding of the NHRPP}

Awareness of the NHRPP was generally low with some participants appearing completely unaware of the programme, while others remembered receiving a letter informing them they were enrolled on the programme but remembered few details. None of the participants could remember receiving an explicit verbal explanation of the programme and its aims from a healthcare professional. However, some recognised that some elements of their care had changed, for example, being appointed a named GP, the GP visiting them to write care plans, being invited into the surgery to review care plans or being informed about the multidisciplinary meeting where the primary care team discussed their care.

Well, this thing came into being, this designated doctor thing came into being maybe a couple of years ago I think. (PT16)

They have a meeting. Once a week, over the surgery. Sort things out, you know? ...Keep things right. (C03)

When you go to see [GP] there, she will say, "[nurse] was saying that you had told her such and such happened", when you go to see [nurse] she'll say "[GP] said such and such". (PT01)

Although participants did not have indepth knowledge of the programme, they were able to discuss their perceptions of the programme and their experience of current care (which itself is the NHRPP). In general, participants were positive about the programme and what they perceived to be its broad objective to provide older people with more care at home and to improve the coordination of services. Many participants thought the programme made sense in that it aimed to support them, or their family member at home and this was in line with their views and wishes.

\section{Well, it sounds a good idea but I don't think that the [GP} practice] could do anymore. (PT15)

I don't want to be in a hospital ward, you know, however nice the people are. You want to keep people in society, not in boxes in some hospital or other. (PT06)

Other participants thought a desire to provide services in a more cost-effective way was the motivation behind the programme and polar opposite views were shared on this perception. This was interpreted positively, as being 'economical' and advantageous to themselves and negatively as being just about reducing costs regardless of the impact on patient care.

C06 I can see the advantages to the NHS if they pursue this, and obviously if there are advantages to the NHS, in a matter of speaking that's huge advantages to us. 
PT06 It's a win, win situation.

But of course you see it ...I know what it's down to. It's all down to cost. I mean it, everything falls to money nowadays and how much things cost. (PT02)

\section{Confidence in the primary healthcare team}

When discussing their recent care, participants voiced high levels of satisfaction with members of the MDT and the level of support provided to them. This support was seen to provide reassurance that their care was being managed and that extra input could be provided if needed. There was a high level of confidence in the members of the primary care team that they could meet patient needs.

I think basically what she's [GP] done is reassured my mum that she's there any time she needs her. And because my mum can't walk to the surgery and back, don't be frightened to call her out. (C05)

I feel like they're looking after us.... (PT13)

As far as the surgery is concerned they're first class. (PT12)

Additionally, participants discussed a high level of satisfaction with access to the MDT. Participants distinguished between telephone access, same-day access and consistent access to one specific professional. Generally, participants were happy to compromise on seeing their preferred professional depending on their need, or to take a telephone consultation rather than a face-to-face appointment. Some patients described being happy to see a nurse who would later liaise with their GP, while others were happy to discuss their care over the telephone.

Or he'll say he'll send the head nurse...to see what's the matter. One of them would be here and see exactly what's the matter and she would confer with him [the $\mathrm{GP}]$ what was to be done. (PT03)

I can, if I'm worried about anything can ring up and I can say, "Would you ask doctor to give me a ring?" and she [GP] always does, and then I'd tell her if I was worried about anything, and if necessary she would come and visit me. (PT10)

One carer felt that same-day access seemed to be better for her husband, who was enrolled on the NHRPP, than herself, who was not enrolled.

But they've usually given you [PT04] a fairly quick appointment haven't they when it's been for you. (C04)

\section{Limitations of home care}

While many participants were satisfied with the care provided for them at home, this was not universally the case. One participant (PT02) felt strongly that there had been occasions, and there may be future occasions, when she thought her needs would best be met in hospital. This participant had respiratory problems and lived alone, she was anxious that despite social care involvement, there were still gaps in her care.

Well, you see there was one point where...I got [the GP] to come over and I said, "Look, I would love to get stabilised..., I would love to go into [hospital]...just to feel as if I was being looked after." I knew that he wasn't going to send me to hospital. (PT02)

To this patient, and others, hospital was not just about meeting medical needs but was about meeting someone's holistic needs whether this was through making them feel safer and therefore, reducing anxiety or by increasing opportunities for social interactions. Other participants discussed their positive past experiences of hospital care and how, although they were generally happy to remain at home, they could envisage circumstances in which they felt that some of their needs may be better met in hospital. This seemed to be magnified by their 'confidence' in local hospitals.

Because in the past two or three years, I've had such a lot go wrong and I've had to go in and out of hospital. I've got a lot of confidence in them. (PT08)

I don't know if you know but [the local hospital] is referred to as the Hilton. (PT09)

I'd rather be in hospital because like I say, I can see people. (PT11)

While, PT02 was resolute that her needs would be best met in hospital, others were usually willing to leave the decision to the primary healthcare team about whether they needed to be admitted to hospital or not.

He [GP] didn't want to send me into hospital, it was that sort of thing....Well I was quite prepared to accept his judgement and the way things went he was right. (PT05)

Participants described how their involvement in care planning led to increased levels of social care and generally, these were well received. However, some participants identified limitations to the level of care provided in terms of providing care at the time needed and sometimes to the extent needed.

Oh they [social carers] are brilliant. I get on really well with them and they do anything for me. (PT13)

The trouble with that was they came to put you to bed at six o'clock at night. I know why, they had to get around a lot of people, but I said "Well, I'm not going to bed...". (PT10)

The lack of overnight care was a commonly mentioned gap in the provision of home care; however, there was a 
noticeable exception. One family member described the short-term provision of overnight sitters on one occasion. In this example, the rapid response team provided overnight care to relieve some of the burden on the family and to support the patient after a recent hospital discharge.

The rapid response team came where they stayed overnight with her just so that she wasn't wandering and I could go home from ten o'clock until eight to sleep. (C16)

The support of family and friends was identified as being vital in terms of filling the gaps in care which could not be met by formal social care. The family member (C16) described how after the one off provision of overnight sitters, the family had managed to provide enough care to keep her mother at home.

These last two [spells of poor health] that she's had close together we've managed to deal with at home. (C16)

The important role played by informal carers was perhaps highlighted most by those patients who did not have someone who could take on an informal caring role. This appeared to increase the perceived benefits of hospital care where 24 hour care was seen to be offered.

I think one of the questions she [the GP] asked me was whether when I was ill if I would go to the hospital, so I said "Well I'd have to because there's nobody to look after me here". (PT10)

I panic...My problems happen at night time, or during the night, when nobody's around. (PT08)

\section{The active role of being a patient}

Participants described how the programme changed not only the way that the primary healthcare team worked but also expectations on themselves to take a more active role in their own care through self-management, care planning and advance care planning. Self-management was well received by patients and was identified by some as promoting independence.

But basically anything that helps self-help, I mean has got to be a good thing and this is what this independence and so forth is about. (PT06)

However, again this was not universally so. PT02 discussed being given a rescue pack for chronic obstructive pulmonary disease and questioning her own judgement about when she should use it.

Now, if I'm left in my own home here with this emergency pack, which is my only thing that I can use, and I would have to use it myself, and I mean they wouldn't be out and in everyday to see if I was all right, so I'd be struggling on my own. (PT02)
Participants discussed their involvement in creating care plans with members of the primary care team. This element of the programme was welcomed by most participants. However, some patients and family members felt that they had not been given enough time to prepare for the care planning and advance care planning discussions; nor was enough time allocated when meeting with the primary care team member to discuss plans. Sometimes, the questions posed were complex and emotive, such as views on resuscitation, or the process was experienced as 'box-ticking' as there was not enough time to make it meaningful to the patient. Other participants felt that the success of their care planning was due to their effort in articulating their views and voiced concerns for other patients who may not be able to take such an active role.

I think it's a good idea and that, but I get nothing out, the year before when I went he didn't think it was necessary to see me. He didn't want to see me. There was nothing I wanted to bring up with him. But overall, I think it's a good idea if you can have a more, have a chat. (PT14)

One of our successes here is that we are both articulate enough to go and ask for these and think about solutions. I do wonder about the ones who have passed beyond that stage, I'm not sure how they would fit into this at all. (C06)

While some participants did suggest amendments to make the process of planning for current and future care more meaningful, others were more content for a less proactive approach. Some participants expressed their views that members of the primary care team were busy and they were happy to contact them on a 'need to' basis.

She probably knows that I'm alright and if I wasn't I'd probably ask for it. (PT07)

I know where they are and if anything goes wrong I go there...he doesn't call to see how I feel because he knows if I want anything I'll be on the blower. (PT15)

\section{DISCUSSION}

The NHRPP was generally well received by participants - patients and carers. Not all participants explicitly recognised that they were enrolled on the NHRPP; however, they were able to discuss their experiences of their current care (which was delivered by the NHRPP). Although they may not have known they were components of the NHRPP, participants discussed their experience of self-management, care planning, advance care planning and feedback from MDT meetings. Perceptions of their care as delivered by the MDT included high levels of satisfaction in terms of their needs being met and access to team members when needed. These findings are in line with other studies 
which have shown satisfaction among patients towards individual case manager-led case management approaches, ${ }^{16} \quad 25-28$ including an appreciation of increased contact with health professionals and greater proactive input, ${ }^{29}{ }^{30}$ and reassurance that care was being coordinated. ${ }^{26} 30$

While the majority of participants had confidence in their primary healthcare team, some raised issues about the limitations of services to replace hospital care. These included the view that hospitals met not just medical needs but psychological needs for social interactions and feelings of safety. This is consistent with current evidence that unmet psychological needs and social isolation are independent predictors for hospital use. ${ }^{31} 32$ Gaps in the current provision of social care were identified, especially for patients who lived alone and did not have anyone who acted as an informal carer. Our results highlight the need to consider further improvement in social care availability. Similar calls have been made in the UK highlighting the need for better integrated primary, community and social care, with services available on a 24 hour basis. ${ }^{33}$

Participants identified the changing nature of the patient role with many of the components of the NHRPP, such as care planning, self-management and encouraging a more active role to be played by patients as opposed to a more traditional and passive patient role. Other studies have shown benefits from patients' experience of enhanced care-planning, ${ }^{34} 35$ including improved self-management capability. ${ }^{36} 37$ If patients are to be fully included in planning their own care, and taking a greater role in self-management of their health, there is a need for time to prepare and carry out such discussions. This supports the findings of a systematic review on personalised care planning which found it was not enough to focus solely on changing practitioner behaviour, but interventions also need to engage patients and provide organisational support. ${ }^{38}$

Our study has added relevance for the NHS in England where the AUAES has been in place since 2014. While we undertook this study in just one English county which may reduce generalisability, Northumberland CCG were forerunners of the AUAES with the NHRPP, and so provides us with a unique group of participants that have had longer to experience case management led by an MDT. A strength of this study is that it includes the views of frail older people who are a hard to reach group. The sample included those with and those without informal carers highlighting some of the gaps that informal carers fill in trying to support older people to remain at home. However, our study has several limitations. Despite sampling a range of practices purposively, our sample of patients was identified by GPs. It is possible that GPs have selected those patients who have had positive experiences of the NHRPP. Future research should seek to purposively sample patients in order to maximise the range of patients included. Another limitation is that participants may have felt inhibited by the presence of another individual where patients and carers were interviewed together. Carers were only included where patients wished this to happen, but carers may have important contributions themselves and further research should investigate their perspective in one-to-one interviews.

Policymakers and clinicians should be aware that our findings suggest that the changes introduced by the NHRPP may be experienced differently by different patients, especially those without informal carers, living alone and with deteriorating health. If further MDT-led case management programmes are to be developed, it is fundamental that patient involvement should be a core component. Further integration of services are needed to allow the complex health, psychological and social needs of frail older people to be addressed in order to reduce unplanned admissions.

Acknowledgements The authors are extremely grateful to the patients and carers who participated in this study, the GP practices who assisted with recruitment and VM for administrative support.

Contributors The study was conceived and designed by RD, AG, CD, TG and LR. Data were collected by $A G$, and analysis was undertaken by $A G, C D, T G$ and $\mathrm{RD}$, with additional analytical support from LR. All authors contributed to the writing of the paper, and have seen and approved the final manuscript. The guarantor of this manuscript is RD.

Funding This work was funded by Northumberland Clinical Commissioning Group. The views expressed are those of the authors and not necessarily those of the Northumberland Clinical Commissioning Group, the NHS or the Department of Health.

Competing interests None declared.

Ethics approval Ethical approval for the study was obtained from National Research Ethics Service Committee Yorkshire and the Humber-Leeds East $(14 / \mathrm{YH} / 1327)$.

Provenance and peer review Not commissioned; externally peer reviewed.

Data sharing statement No additional data are available.

Open Access This is an Open Access article distributed in accordance with the Creative Commons Attribution Non Commercial (CC BY-NC 4.0) license, which permits others to distribute, remix, adapt, build upon this work noncommercially, and license their derivative works on different terms, provided the original work is properly cited and the use is non-commercial. See: http:// creativecommons.org/licenses/by-nc/4.0/

\section{REFERENCES}

1. United Nations. Department of economic and social affairs. Population division. World population ageing 2013. New York NY: United Nations, Department of Economic and Social Affairs, Population Division, 2013

2. Office for National Statistics. Population trends. 142 edn. London, UK 2010.

3. van den Akker M, Buntinx F, Metsemakers JF, et al. Multimorbidity in general practice: prevalence, incidence, and determinants of co-occurring chronic and recurrent diseases. J Clin Epidemiol 1998;51:367-75.

4. Clegg A, Young J, lliffe S, et al. Frailty in elderly people. Lancet 2013;381:752-62

5. National Audit Office (NAO). Emergency admissions to hospital: managing the demand. London, UK: The Stationary Office (TSO), 2013:49.

6. World Health Organisation. World report on ageing and health. Geneva, Switzerland, 2015.

7. Wodchis WP, Dixon A, Anderson GM, et al. Integrating care for older people with complex needs: key insights and lessons from a seven-country cross-case analysis. Int J Integr Care 2015;15:e021. 
8. Clarkson P, Brand C, Hughes J, et al. Integrating assessments of older people: examining evidence and impact from a randomised controlled trial. Age Ageing 2011;40:388-91.

9. Gröne O, Garcia-Barbero M, WHO European Office for Integrated Health Care Services. Integrated care: a position paper of the WHO European Office for Integrated Health Care Services. Int J Integr Care 2001;1:e21.

10. Ross S, Curry N, Goodwin N. Case management. What is it and how can it best be implemented? London, UK: The King's Fund, 2011.

11. Purdy S, Paranjothy S, Huntley A, et al. Interventions to reduce unplanned hospital admission: a series of systematic reviews. Bristol, UK: National Institute for Health Research, 2012.

12. Low LF, Yap M, Brodaty $H$. A systematic review of different models of home and community care services for older persons. BMC Health Serv Res 2011;11:93.

13. You EC, Dunt D, Doyle C, et al. Effects of case management in community aged care on client and carer outcomes: a systematic review of randomized trials and comparative observational studies. BMC Health Serv Res 2012;12:395

14. Hutt R, Rosen R, McCauley J. Case-managing long-term conditions: what impact does it have on the treatment of older people? London, UK: The King's Fund, 2004.

15. Eklund K, Wilhelmson K. Outcomes of coordinated and integrated interventions targeting frail elderly people: a systematic review of randomised controlled trials. Health Soc Care Community 2009;17:447-58.

16. Stokes J, Panagioti M, Alam R, et al. Effectiveness of case management for 'at risk' patients in primary care: a systematic review and meta-analysis. PLOS ONE 2015;10:e0132340.

17. Humphries R. Integrated health and social care in Englandprogress and prospects. Health Policy 2015;119:856-9.

18. Stokes J. CCG implementation of integrated care in the NHS. BMC Health Serv Res 2014;14:P119.

19. Stokes J, Kristensen SR, Checkland K, et al. Effectiveness of multidisciplinary team case management: difference-in-differences analysis. BMJ Open 2016;6:e010468.

20. Craig P, Dieppe P, Macintyre S, et al, Medical Research Council Guidance. Developing and evaluating complex interventions: the new Medical Research Council guidance. BMJ 2008;337:a1655.

21. NHS England. Enhanced service specification. Avoiding Unplanned Admissions: proactive case finding and patient review for vulnerable people, 2014

22. Alan B. Social research methods. London, UK: Oxford University Press, 2008.

23. Richards H, Emslie C. The 'doctor'or the 'girl from the University'? Considering the influence of professional roles on qualitative interviewing. Fam Pract 2000;17:71-5.
24. Braun V, Clarke V. Using thematic analysis in psychology. Qual Res Psychol 2006;3:77-101.

25. Leighton $Y$, Clegg A, Bee A. Evaluation of community matron services in a large metropolitan city in England. Qual Prim Care 2008;16:83-9.

26. Sargent P, Pickard S, Sheaff R, et al. Patient and carer perceptions of case management for long-term conditions. Health Soc Care Community 2007;15:511-19.

27. Williams V, Smith A, Chapman L, et al. Community matrons-an exploratory study of patients' views and experiences. J Adv Nurs 2011;67:86-93.

28. Hudon C, Chouinard MC, Diadiou F, et al. Case management in primary care for frequent users of health care services with chronic diseases: a qualitative study of patient and family experience. Ann Fam Med 2015;13:523-8.

29. Sheaff R, Boaden R, Sargent $P$, et al. Impacts of case management for frail elderly people: a qualitative study. J Health Serv Res Policy 2009;14:88-95.

30. Sandberg M, Jakobsson U, Midlöv $P$, et al. Case management for frail older people-a qualitative study of receivers' and providers' experiences of a complex intervention. BMC Health Serv Res 2014;14:14

31. Geller J, Janson P, McGovern E, et al. Loneliness as a predictor of hospital emergency department use. J Fam Pract 1999;48:801-4

32. Quail JM, Wolfson C, Lippman A. Unmet need and psychological distress predict emergency department visits in communitydwelling elderly women: a prospective cohort study. BMC Geriatr 2011;11:86

33. British Geriatrics Society. Fit for frailty: part 2: developing, commissioning and managing services for people living with frailty in community settings. London, UK: British Geriatrics Society and the Royal College of General Practitioners with Age UK, 2015.

34. Burt J, Roland M, Paddison C, et al. Prevalence and benefits of care plans and care planning for people with long-term conditions in England. J Health Serv Res Policy 2012;17(Suppl 1):64-71.

35. Bodenheimer T, Wagner EH, Grumbach K. Improving primary care for patients with chronic illness. JAMA 2002;288:1775-9.

36. Gibson PG, Powell H, Coughlan J, et al. Self-management education and regular practitioner review for adults with asthma. Cochrane Database Syst Rev 2003;(1):CD001117.

37. Coulter A, Entwistle VA, Eccles A, et al. Personalised care planning for adults with chronic or long-term health conditions. Cochrane Database Syst Rev 2015;(3):CD010523.

38. Graffy J, Eaton S, Sturt J, et al. Personalized care planning for diabetes: policy lessons from systematic reviews of consultation and self-management interventions. Prim Health Care Res Dev 2009;10:210-22. 